
\title{
28 Research Square \\ Comparison of the efficacy of five front-of-pack nutrition labels in helping the Brazilian consumer make a healthier choice
}

Wendy A.M. Blom ( $\square$ Wendy.Blom@unilever.com )

Unilever Innovation Vlaardingen https://orcid.org/0000-0003-2591-6958

Chantal Goenee

Unilever Innovation Vlaardingen

Lucia Juliano

Harris Interactive UK Ltd

Els M. de Groene

Unilever Foods Innovation Center, Wageningen

Fernanda de Oliveira Martins

Unilever https://orcid.org/0000-0002-4129-9526

\section{Research article}

Keywords: online consumer research, front-of-pack label, Nutri-Score, warning label, traffic light label, Brazil

Posted Date: October 6th, 2020

DOI: https://doi.org/10.21203/rs.3.rs-71579/v1

License: (c) (1) This work is licensed under a Creative Commons Attribution 4.0 International License. Read Full License

Version of Record: A version of this preprint was published at Food Science \& Nutrition Research on December 30th, 2021. See the published version at https://doi.org/10.33425/2641-4295.1045. 


\section{Abstract \\ Background:}

We tested, in an online survey, how well five different front-of-pack (FOP) labels helped Brazilian consumers make a healthier choice between two food or beverage products as compared to a no FOP label control.

\section{Methods:}

All 1072 respondents were randomly assigned to one of six groups 1) no FOP label (control), 2) ABIA label, 3) GGALIi Nutrient Profile label, 4) IdeC label, 5) Hybrid label or 6) Nutri-Score label and were all shown 9 food stimuli consisting of two products. The nutrient profile of the ABIA and Hybrid labels take into account the serving size of the food, while the other three labels score per $100 \mathrm{~g}$. Respondents were asked which of the two products they thought was the healthier choice.

\section{Results:}

Overall, the Hybrid and ABIA labels performed best, resulting in a statistically significantly higher percentage of correct answers compared to the control for $9 / 9$ and $8 / 9$ of the food stimuli, respectively. Nutri-Score performed reasonably well and outperformed the control in 6/9 cases. The IdeC and GGALIi NP warning labels were the least useful, performing only one and two times better, respectively, than the control group.

\section{Conclusion:}

The Hybrid and the ABIA FOP labels, two interpretative traffic light labels that use colours and provide nutritional information per serving, were best suited to help Brazilian consumers choose the healthier product. They especially outperformed the other FOP labels when serving sizes differed significantly or when deeper consideration of nutritional information was needed to make an informed decision.

\section{Background}

Although many countries and health organizations have issued dietary guidelines with clear recommendations as to which food groups fit into a healthy diet and which foods and nutrients should be consumed in moderation, adherence to dietary recommendations is low.

Nutrient declarations on the back of the pack provide important information about the nutritional composition of food products, but consumers find this information complex and difficult to understand [1, 2]. That's why, Front-of-Pack (FOP) nutrition labelling systems are designed to help consumers make a quick, informed decision about the nutritional content or relative healthfulness of a food or beverage.

There is no international standardization of FOP labels and therefore many different FOP labels have been developed and implemented around the world [3-5]. These FOP labels differ not only in graphical 
presentation, but also in the type and level of information they provide, the nutritional profiles used, the food products covered and the implementation (i.e. voluntary or mandatory). This is at least partly explained by the fact that the model must meet the needs of each country, taking into consideration cultural and dietary patterns, education level and alignment with national dietary guidance.

FOP labels can be roughly divided into two types namely interpretative and non-interpretative labels. Noninterpretative FOP labels provide information on the amount of key nutrients (e.g. fat, sugar and sodium) and percent recommended intakes, with little interpretation of this information (e.g. Guideline Daily Amounts [GDA] or Reference Intake [RI] logos [6]). Interpretative FOP labels contain an interpretation of the nutritional quality of the products, with or without information about the amount of key nutrients. Some FOP labels use colours (e.g. Multiple Traffic Light [MTL] [7]) to indicate whether the level of a nutrient is high (red), medium (amber) or low (green). Other interpretive FOP labels provide a simple summary score of a product's overall nutritional profile (e.g. Keyhole logo [8], Choices Programme Logo [9]), a ranking (e.g. Health Star Rating [10], Nutri-Score [11]) or warn for high levels of certain critical nutrients in products (e.g. Warning labels [12]).

Many studies have examined the different attributes of effectiveness of FOP labels, but there are numerous inconsistencies in the results. This can be explained by the fact that the definition of effectiveness differs from study to study. Some studies focus on consumer liking, understanding or preference for FOP labels, others on food choice or actual or intended food purchase and some on food intake. There are also major differences in methodologies used. However, research into the effectiveness of FOP labels on consumer behaviour in practise is lacking [13].

Studies focusing on consumers' understanding of FOP labels and product choice generally show that FOP labels appear to help consumers determine which foods are healthier and which are less healthy $[14,15]$. Simple FOP labels such as MTL, warning labels and Nutri-Score appear to be most effective [15].

The MTL label has been implemented in the United Kingdom [7]. It provides information on energy, fat, saturated fat, sugar and salt content per 100 gram and as percentage of reference intake, combined with traffic lights colours (green, amber and red) to highlight low, medium or high levels of the nutrients. It also provides serving size information that is expressed in easily recognizable and meaningful ways to the consumer (e.g. $1 / 4$ of a pie). Nutri-Score is an interpretive FOP label that uses letters and colours to rank healthiness of products [11]. The French government adopted the Nutri-Score in 2017 and since then governments of other countries such as Belgium, Switzerland and Germany have also chosen to adopt the Nutri-Score $[16,17]$. In Latin America, the implementation of warning labels is spreading. Ecuador was the first to implement a mandatory FOP label system, namely a traffic light system [18]. They were followed by Chile which implemented mandatory warning labels in 2016 [19]. Since then, Peru [20], Paraguay and Uruguay have also decided to implement warning labels and Mexico has recently followed suit [21]. Brazil is currently reviewing mandatory nutrition labelling. Anvisa, the National Health Surveillance Agency of Brazil established a working group on Nutrition Labelling to identify problems in the transmission of nutritional information and alternatives that can help improve the effectiveness of nutrition labelling [22]. Several FOP labels were proposed to Anvisa and reviewed. 
This study was designed to determine which of five different types of nutritional front-of-pack labels best helps Brazilian respondents identify the healthiest choice between two food products, compared to a nonlabel control. Four of the tested FOP labels were also reviewed by Anvisa. We wanted to test the robustness of the efficacy of different FOP labels by also comparing products from different product categories, consumed in different serving sizes or with closer nutritional profiles. As a result, a wide variety of products were tested.

\section{Methods}

\subsection{Study population}

Study participants were recruited from an existing research panel of Brazilian consumers that represent general members of the public. The aim was to have a representative sample of respondents and an even gender split. Participants were eligible for participation if they were aged between 18 and 65 years.

\subsection{Front-of-pack labels}

Five FOP labels were tested in this study, i.e. GGALIi Nutrient Profile, IdeC, ABIA, Nutri-Score and a hybrid label which was developed for this study. The first four FOP labels were selected because they were proposed by various stakeholders to Anvisa, the National Health Surveillance Agency of Brazil [22]. The selected labels cover three different visual expressions of nutritional labelling. GGALIii Nutrient Profile and IdeC labels are warning style labels, Nutri-Score is an interpretative colour coded label. The ABIA label is a traffic light system that provides nutritional information. The hybrid label is an adaptation of the Evolved Nutrition Label [23] and contains nutritional information as well as a red colour code, as warning sign, if levels of an ingredient are high. The Control Group received visual expressions of products without a FOP label. This group was used as a reference.

This study focused on the three nutrients of concern that were initially proposed by Anvisa, i.e. saturated fat, sugar and sodium [22]. Hence other nutrients were not taken into account.

GGALI ii NP label: GGALI (Gerência-Geral de Alimentos) is Anvisa's General Food Management who prepared the "regulatory impact analysis" published by Anvisa in 2018 [22]. GGALI proposed two nutrient profiles. We selected the stricter one - GGALIi - which was later chosen by Anvisa. The GGALIi NP label is a warning style label highlighting high levels of nutrients of concern. It is based on the nutrient content per $100 \mathrm{~g}$ or $100 \mathrm{ml}$ for food and beverages, having as reference the guidelines of the World Health Organization (WHO) and Codex Alimentarius [22]. Criteria were defined for low, medium and high content of free sugars, saturated fat, total fat and sodium [22]. For the GGALIi NP label in this study criteria for high levels were applied. When this study was designed, Anvisa had not chosen a visual model. We used the magnifying glass visual that was also considered by Anvisa, and in the end also selected. This visual is also under discussion in Canada.

IDEC label: IDEC (Instituto Brasileiro de Defesa do Consumidor) is a civil society in Brazil. They proposed a FOP label that is a warning style label and uses black triangles to inform the high content of sugars, total fat, saturated fat and sodium, and the presence of trans fats and sweeteners. The nutritional profile model was 
adapted from the Pan American Health Organization (PAHO) profile model and is based on percentage of energy [22].

ABIA label: ABIA (Associação Brasileira das Indústrias da Alimentação), representing the Brazilian food industry sector, proposed a Multiple Traffic Light (MTL) FOP label, based on the United Kingdom traffic lights, which reports the absolute quantities of sugars, saturated fats and sodium per serving. It uses the red, amber and green colours to indicate the high, medium and low levels of each nutrient according to criteria per serving [22].

Nutri-Score: Nutri-Score is an interpretative, graded, colour-coded FOP label that has been developed by French researchers [11]. It is based on the nutrient profiling system of the United Kingdom Food Standards Agency which uses the nutrient content per $100 \mathrm{~g}$ for food and beverages. Positive points (0-10) are allocated for energy, total sugar, saturated fat and sodium content and negative points (0-5) are allocated for fruit, vegetables and nuts, fibre and protein content. Products scores range from - 15 (most healthy) to + 40 (least healthy) [11] and are translated into five categories of nutritional quality ranging from $A$ (green) to $E$ (red).

Hybrid label: The hybrid FOP label is a model developed for this study and is an adaptation of the Evolved Nutrition Label (ENL) [23]. Criteria for sugar, saturated fat and sodium were defined for small serving sizes (< $60 \mathrm{~g})$, medium serving sizes (60-120 g) and large serving sizes (> $120 \mathrm{~g}$ ). In line with ENL, calculations were done per serving size, except for serving sizes between 60 and $120 \mathrm{~g}$, in which case calculations were done per $100 \mathrm{~g}$. The label provides quantitative nutritional information per serving and uses the red colour to indicate high amounts of the nutrient in a serving of the product.

An example of the five labels is provided in Fig. 1. The specific criteria used for the ABIA, GGALIii, IdeC and Hybrid labels are presented in Table 1. For the Nutri-Score label, the Nutri-Score algorithm was used to calculate the score for each product [11]. 
Table 1

Criteria used for the ABIA, GGALIi NP, IdeC and Hybrid labels

\begin{tabular}{|c|c|c|c|c|c|c|}
\hline Label & Product criteria & Category & $\begin{array}{l}\text { Total sugar } \\
\text { (g) }\end{array}$ & $\begin{array}{l}\text { Free } \\
\text { sugars } \\
\text { (g) }\end{array}$ & $\begin{array}{l}\text { Saturated } \\
\text { fat (g) }\end{array}$ & $\begin{array}{l}\text { Sodium } \\
\text { (mg) }\end{array}$ \\
\hline ABIA label & $\begin{array}{l}\text { Solids }<100 \mathrm{~g} \text { and } \\
\text { Liquids }(\mathrm{ml})\end{array}$ & Low & $\leq 5,0$ & & $\leq 1,5$ & $\leq 80$ \\
\hline ABIA label & $\begin{array}{l}\text { Solids }<100 \mathrm{~g} \text { and } \\
\text { Liquids }(\mathrm{ml})\end{array}$ & Medium & $>5,0 \leq 13,5$ & & $>1,5 \leq 3,0$ & $>80 \leq 300$ \\
\hline ABIA label & $\begin{array}{l}\text { Solids }<100 \mathrm{~g} \text { and } \\
\text { Liquids }(\mathrm{ml})\end{array}$ & High & $>13,5$ & & $>3,0$ & $>300$ \\
\hline ABIA label & Solids $\geq 100 \mathrm{~g}$ & Low & $\leq 5,0$ & & $\leq 1,5$ & $\leq 80$ \\
\hline ABIA label & Solids $\geq 100 \mathrm{~g}$ & Medium & $>5,0 \leq 27,0$ & & $>1,5 \leq 6,0$ & $>80 \leq 600$ \\
\hline ABIA label & Solids $\geq 100 \mathrm{~g}$ & High & $>27,0$ & & $>6,0$ & $>600$ \\
\hline $\begin{array}{l}\text { GGALIi } \\
\text { NP label }\end{array}$ & Solid (100 g) & $\begin{array}{l}\text { Warning } \\
\text { label }\end{array}$ & & $\geq 10$ & $\geq 4$ & $\geq 400$ \\
\hline $\begin{array}{l}\text { GGALIi } \\
\text { NP label }\end{array}$ & Liquids (100 mL) & $\begin{array}{l}\text { Warning } \\
\text { label }\end{array}$ & & $\geq 5$ & $\geq 2$ & $\geq 200$ \\
\hline $\begin{array}{l}\text { IdeC } \\
\text { label\# }\end{array}$ & All products & $\begin{array}{l}\text { Warning } \\
\text { label }\end{array}$ & & $\geq 10 \% \mathrm{E}$ & $\geq 10 \% \mathrm{E}$ & $\geq_{1 \mathrm{mg} / \mathrm{kcal}}$ \\
\hline $\begin{array}{l}\text { Hybrid } \\
\text { label }\end{array}$ & $\begin{array}{l}\text { Solids }<60 \mathrm{~g} \text { and } \\
\text { Liquids }\end{array}$ & High & $\begin{array}{l}>13,5 \text { per } \\
\text { serving }\end{array}$ & & $\begin{array}{l}>3,0 \text { per } \\
\text { serving }\end{array}$ & $\begin{array}{l}>300 \text { per } \\
\text { serving }\end{array}$ \\
\hline $\begin{array}{l}\text { Hybrid } \\
\text { label }\end{array}$ & $\begin{array}{l}\text { Solids } \geq 60 \mathrm{~g} \leq \\
120 \mathrm{~g}\end{array}$ & High & $\begin{array}{l}>22,5 \text { per } \\
100 \mathrm{~g}\end{array}$ & & $\begin{array}{l}>5,0 \text { per } \\
100 \mathrm{~g}\end{array}$ & $\begin{array}{l}>500 \text { per } \\
100 \mathrm{~g}\end{array}$ \\
\hline $\begin{array}{l}\text { Hybrid } \\
\text { label }\end{array}$ & Solids > $120 \mathrm{~g}$ & High & $\begin{array}{l}>27 \text { per } \\
\text { serving }\end{array}$ & & $\begin{array}{l}>6,0 \text { per } \\
\text { serving }\end{array}$ & $\begin{array}{l}>600 \text { per } \\
\text { serving }\end{array}$ \\
\hline
\end{tabular}

\subsection{Food stimuli}

This study included 18 food items, which were presented to the respondents in sets of two. The respondents were asked to indicate which of the two products they thought was healthier. The correct answer was defined considering the contents of the three nutrients highlighted on the front of pack label. That is, the product with the lowest sugar, saturated fat and sodium content per serving was considered the healthiest choice. When a product was higher in one nutrient and lower in another, the larger difference was considered most important. In a few cases, one nutrient was slightly lower and one much higher. Subsequently the much higher nutrient was considered more important for the classification.

The food sets (food stimuli) were carefully selected to test the robustness of the labels to help the consumer identify the healthier option. The food stimuli differed with regard to the following variables: similar products consumed in small serving size, similar products consumed in large serving size, similar product but 
consumed in different serving sizes, and products from different food categories but consumed in same eating occasion. The food categories included in the study were soft cheeses, fat spreads, ice creams, lasagne, frozen meals, fermented milks \& chocolate oat drinks, sweet snacks, cereal bars \& yoghurt and chocolate bars. The products corresponded to different consumption occasions (e.g. breakfast, lunch or main meal and in-between meal snack). Figure 2 shows an example of one of the stimuli as presented to the respondents. In Brazil it is not mandatory to declare sugar content of food products on the packaging. For some of the products used in this study, i.e. ice creams, frozen meals, the dairy alternative drink, and sweet snacks, we had to estimate the sugar content. Estimations were based on similar products marketed in countries where sugar content is declared in the nutrition table. Table 2 summarizes the nutritional profiles of the food stimuli. 
Table 2

Nutritional profile of food stimuli

\begin{tabular}{|c|c|c|c|c|c|c|c|c|}
\hline & & & Per sen & & & Per 10 & & \\
\hline $\begin{array}{l}\text { Food } \\
\text { category }\end{array}$ & Product & $\begin{array}{l}\text { Serving } \\
\text { size (g) }\end{array}$ & $\begin{array}{l}\text { Sugar } \\
\text { (g) }\end{array}$ & $\begin{array}{l}\text { Saturated } \\
\text { fat }(\mathrm{g})\end{array}$ & $\begin{array}{l}\text { Sodium } \\
\text { (mg) }\end{array}$ & $\begin{array}{l}\text { Sugar } \\
\text { (g) }\end{array}$ & $\begin{array}{l}\text { Saturated } \\
\text { fat }(g)\end{array}$ & $\begin{array}{l}\text { Sodium } \\
\text { (mg) }\end{array}$ \\
\hline Soft Cheese & $\begin{array}{l}\text { Product } \\
1^{\star}\end{array}$ & 30 & NA & 1,9 & 118 & NA & 6,3 & 393 \\
\hline Soft Cheese & $\begin{array}{l}\text { Product } \\
2\end{array}$ & 30 & NA & 5,2 & 239 & NA & 17,3 & 796 \\
\hline Fat spreads & $\begin{array}{l}\text { Product } \\
1^{*}\end{array}$ & 10 & NA & 0,9 & 70 & NA & 9 & 700 \\
\hline Fat spreads & $\begin{array}{l}\text { Product } \\
2\end{array}$ & 10 & NA & 4,8 & 90 & NA & 48 & 900 \\
\hline Ice cream & $\begin{array}{l}\text { Product } \\
1\end{array}$ & 86 & 21,5 & 7,7 & 40 & 25 & 8,9 & 46,5 \\
\hline Ice cream & $\begin{array}{l}\text { Product } \\
2^{*}\end{array}$ & 60 & 13 & 1,8 & 12 & 21,7 & 3 & 20 \\
\hline Lasagne & $\begin{array}{l}\text { Product } \\
1\end{array}$ & 400 & 12 & 10 & 1280 & 3 & 2,5 & 320 \\
\hline Lasagne & $\begin{array}{l}\text { Product } \\
2^{\star}\end{array}$ & 400 & 11,2 & 5,2 & 1440 & 2,8 & 1,3 & 360 \\
\hline $\begin{array}{l}\text { Frozen } \\
\text { meals }\end{array}$ & $\begin{array}{l}\text { Product } \\
1^{*}\end{array}$ & 300 & NA & 2,9 & 250 & NA & 0,9 & 83,3 \\
\hline $\begin{array}{l}\text { Frozen } \\
\text { meals }\end{array}$ & $\begin{array}{l}\text { Product } \\
2\end{array}$ & 275 & NA & 9,3 & 1242 & NA & 3,4 & 451,6 \\
\hline $\begin{array}{l}\text { Fermented } \\
\text { milk drink }\end{array}$ & $\begin{array}{l}\text { Product } \\
1\end{array}$ & 200 & 32,5 & 0 & 75 & 16,25 & 0 & 37,5 \\
\hline $\begin{array}{l}\text { Chocolate } \\
\text { oat drink }\end{array}$ & $\begin{array}{l}\text { Product } \\
2^{\star}\end{array}$ & 260 & 12 & 0,6 & 60 & 4,6 & 0,2 & 23 \\
\hline $\begin{array}{l}\text { Sweet } \\
\text { snacks }\end{array}$ & $\begin{array}{l}\text { Product } \\
1\end{array}$ & 40 & 10,6 & 3,4 & 68 & 26,6 & 8,4 & 170 \\
\hline $\begin{array}{l}\text { Sweet } \\
\text { snacks }\end{array}$ & $\begin{array}{l}\text { Product } \\
2^{\star}\end{array}$ & 20 & 6,6 & 2,1 & 26 & 33 & 10,5 & 130 \\
\hline Cereal Bar & $\begin{array}{l}\text { Product } \\
1^{*}\end{array}$ & 21 & 6,9 & 0,8 & 0 & 32,8 & 3,8 & 0 \\
\hline Yoghurt & $\begin{array}{l}\text { Product } \\
2\end{array}$ & 170 & 20,4 & 4,6 & 160 & 12 & 2,7 & 94 \\
\hline $\begin{array}{l}\text { Chocolate } \\
\text { bars }\end{array}$ & $\begin{array}{l}\text { Product } \\
1^{*}\end{array}$ & 16,7 & 8 & 2,6 & 16 & 47,9 & 15,5 & 100 \\
\hline
\end{tabular}




\begin{tabular}{|c|c|c|c|c|c|c|c|c|}
\hline \multirow[b]{2}{*}{$\begin{array}{l}\text { Chocolate } \\
\text { bars }\end{array}$} & \multirow[b]{2}{*}{$\begin{array}{l}\text { Product } \\
2\end{array}$} & \multirow[b]{2}{*}{40} & \multicolumn{3}{|c|}{ Per serving } & \multicolumn{3}{|c|}{ Per $100 \mathrm{~g}$} \\
\hline & & & 19,9 & 6,6 & 40 & 49,8 & 16,5 & 100 \\
\hline
\end{tabular}

\subsection{Data collection}

A 5-minute online survey was conducted in July 2019, using Toluna QuickSurveys. All respondents were randomly allocated to one of six groups 1) no label (control), 2) ABIA label, 3) GGALIi NP label, 4) IdeC label, 5) Hybrid label or 6) Nutri-Score label.

Apart from the control group, the respondents were shown the FOP label. The FOP label was briefly explained. The respondents were then shown 9 food stimuli consisting of two products and, based on the information they received, they were asked which product they thought was the healthier choice. The respondents in the control group were also shown the same 9 choice sets of products, but without a label. Respondents were provided with four potential answers: 1) Product 1; 2) Product 2; 3) No difference and 4) I don't know. Respondents were also asked to rate, on a scale from 0 to 10 , how useful the label was in helping them make a healthy food choice and what they liked and disliked about the specific type of label that they had evaluated.

\subsection{Statistical analyses}

Descriptive statistics were calculated for the sociodemographic data of the participants. The percentage of participants selecting the answers "Product 1", "Product 2", "No difference" or "Don't know" were calculated for each set of food stimuli, for each FOP label group as well as for the control group. Significance testing (Ztest) was performed to test if the proportion of participants correctly identifying the healthier product differed between the FOP label groups. Significance tests were performed within these subgroups to test whether participants who correctly chose the healthier option differed according to education level or income. The mean scores for usefulness of the labels were calculated. T-tests were used to test for statistical differences between mean scores.

Statistics were performed with the Toluna Analytics tool. A significance testing at a $95 \%$ confidence level was used.

\section{Results}

\subsection{Respondents}

A total of 1072 Brazilian men and women participated in the online survey. Sociodemographic data are presented in Table 3. A total of 176 respondents were included in the control group, 181 respondents were allocated to the ABIA group, 177 respondents were allocated to the GGALIi NP warning label, 181 
respondents to the IdeC - triangle warning label, 178 respondents to the hybrid colour code label and 179 respondents were allocated to the Nutri-Score label.

There were no consistent significant differences in terms of education and income levels between the different groups. 
Table 3

Sociodemographic data

\section{Characteristics}

\begin{tabular}{|lc|}
\hline Sex & Number of participants (\%) \\
\hline Female & $578(54 \%)$ \\
\hline Male & $494(46 \%)$ \\
\hline Age & \\
\hline $18-34$ & $681(64 \%)$ \\
\hline $35-54$ & $338(32 \%)$ \\
\hline $55+$ & $53(5 \%)$ \\
\hline
\end{tabular}

\section{Education}

\begin{tabular}{|c|c|}
\hline Low & $82(8 \%)$ \\
\hline Medium & $481(45 \%)$ \\
\hline High & $496(46 \%$ \\
\hline Undisclosed & $13(1 \%)$ \\
\hline \multicolumn{2}{|l|}{ Social grade } \\
\hline$A$ & 209 (19\%) \\
\hline B1 & $194(18 \%)$ \\
\hline B2 & $329(31 \%)$ \\
\hline C1 & $179(17 \%)$ \\
\hline $\mathrm{C} 2$ & $103(10 \%)$ \\
\hline$D-E$ & $22(2 \%)$ \\
\hline Undisclosed & $36(3 \%)$ \\
\hline \multicolumn{2}{|l|}{ Income } \\
\hline Low & $596(56 \%)$ \\
\hline High & $401(37 \%)$ \\
\hline Undisclosed & $75(7 \%)$ \\
\hline \multicolumn{2}{|c|}{ Geographic location } \\
\hline Midwest & $60(6 \%)$ \\
\hline Northeast & $227(21 \%)$ \\
\hline
\end{tabular}




\begin{tabular}{|ll|}
\hline Characteristics \\
\hline North & $41(4 \%)$ \\
\hline South & $145(14 \%)$ \\
\hline Southeast & $581(54 \%)$ \\
\hline Undisclosed & $18(2 \%)$ \\
\hline Household shopping responsibility & \\
\hline Sole & $54 \%$ \\
\hline Joint & $38 \%$ \\
\hline None & $8 \%$ \\
\hline
\end{tabular}

\subsection{Accuracy of choosing the healthier product}

Table 4 summarizes for each of the labels and the control group the percentages of respondents that correctly identified the healthier product for each of 9 stimuli.

When the soft cheese food stimuli were presented, most respondents accurately identified the healthier product. Respondents who were shown the IdeC warning label performed significantly worse than the control group; a third of the respondents in this group indicated that there was no difference between the two products and only $51 \%$ of the respondents correctly identified the healthier product.

Respondents who were shown the ABIA or hybrid labels were most likely to identify the healthier choice from the fat spreads category. Most respondents who were shown the GGALI ii or IdeC warning style labels selected the least healthy product of the two ( $41 \%$ and $44 \%$, respectively) or indicated that there was no difference between the two products ( $32 \%$ and $24 \%$, respectively). The GGALI Iabel scored worse than the control group. Also $55 \%$ of the respondents in the control group chose the less healthy product.

For the ice cream stimuli, the group of respondents who were shown the IdeC labels had most difficulty choosing the healthier option; only $13 \%$ of the respondents chose the healthier product. Forty percent of the respondents in the control group and $60 \%$ of the respondents from the IdeC group indicated that there was no difference between the two ice cream products. The ABIA and hybrid labels best helped respondents to make the healthier choice: $85 \%$ and $88 \%$ respectively chose the healthier product.

Many respondents had difficulty identifying the healthier lasagne. Only the respondents who were shown the hybrid label performed significantly better ( $64 \%$ correct) than the control group ( $48 \%$ correct). The Nutri-Score label only helped $20 \%$ of the respondents make the right choice, compared to $48 \%$ of the respondents in the control group. Seventy percent of the respondents who were shown the Nutri-Score label indicated that there was no difference between the products.

Respondents in the control group and those shown the IdeC label found it particularly difficult to choose the healthier option from the frozen meals. A total of $56 \%$ of the respondents in the control group and $62 \%$ of 
respondents in the IdeC label group indicated that there was no difference between the two frozen meals. The $\mathrm{ABI}$ and hybrid label performed best.

In the fermented milk and chocolate oat drinks category, the ABIA, Hybrid and Nutri-Score labels performed best and the GGALI ii and IdeC labels performed worse: about a third of respondents chose the less healthy option and about a third indicated that there is no difference between products.

There was a marked difference between labels in their ability to help choose the healthier sweet snack. The majority of the respondents in the control group (56\%) and those who were shown the GGALIi $(73 \%)$ or Idec (57\%) label considered that there was no difference between the two products with regard to health. The labels ABIA, Hybrid and Nutri-Score performed significantly better.

When respondents were shown a cereal bar and a yoghurt product, i.e. two very different products, the $A B I A$, IdeC and hybrid labels were the most successful in helping them make a healthier choice. Forty-five percent of respondents who were shown the GGALIi label believed that there was no difference between products and $72 \%$ of respondents who were shown the Nutri-Score label chose the less healthy product.

There was confusion amongst the majority of the respondents for the chocolate bar category (showing two different sizes of the same brand chocolate bar). Most respondents seeing the GGALI ${ }^{\mathrm{ii}}(59 \%)$, Idec (56\%) or Nutri-score (63\%) labels, which do not consider serving size, thought that there was no difference between the two products. The ABIA and Hybrid labels, which do consider serving size, resulted in the highest numbers of respondents choosing the healthier option.

Overall, the IdeC warning label was least helpful for consumers to make the healthier choice. Eight out of nine times, the IdeC label for the healthier product was the same as for the less healthy product, providing no guidance to the consumer. Only once, when comparing the cereal bar and yoghurt, the IdeC label outperformed the control group. In that case, $70 \%$ of the respondents chose the healthier option. The other warning label, GGALI ${ }^{\mathrm{ii}} \mathrm{NP}$, performed a bit better than the IdeC label, but also failed to distinguish products six out of nine times. It outperformed the control group in only two out of nine cases. Nutri-Score performed reasonably well but also failed two times in guiding the consumer to the healthier choice when products were given the same rating. This happened for example when the serving sizes of the two products differed significantly. In case of the cereal bar $(21 \mathrm{~g})$ versus yoghurt (170 g) food stimulus, a better Nutri-Score was given for the least healthy product (yoghurt).

The hybrid label performed best, resulting in statistically significantly higher percentage of correct answers as compared to the control in all cases. The ABIA label outperformed the control group eight times out of nine. 
Table 4

Percentage of participants correctly choosing the healthiest option within each food stimulus, by randomization assignment to FOP label or control

\begin{tabular}{|c|c|c|c|c|c|c|c|c|c|}
\hline & $\begin{array}{l}\text { Soft } \\
\text { cheese }\end{array}$ & $\begin{array}{l}\text { Fat } \\
\text { spreads }\end{array}$ & $\begin{array}{l}\text { lce } \\
\text { cream }\end{array}$ & Lasagne & $\begin{array}{l}\text { Frozen } \\
\text { meals }\end{array}$ & $\begin{array}{l}\text { Milk } \\
\text { drinks }\end{array}$ & $\begin{array}{l}\text { Sweet } \\
\text { snacks }\end{array}$ & $\begin{array}{l}\text { Cereal } \\
\text { bar \& } \\
\text { yoghurt }\end{array}$ & $\begin{array}{l}\text { Chocolate } \\
\text { bars }\end{array}$ \\
\hline Control & $76^{d}$ & $37^{c}$ & $31^{d}$ & $48^{d, f}$ & 13 & 40 & $26^{c}$ & $44^{c, f}$ & $39 c, f$ \\
\hline ABIA & $88^{a, c, d}$ & $82^{a, c, d, f}$ & $\begin{array}{l}85 \\
a, c, d, f\end{array}$ & $46^{d, f}$ & $\begin{array}{l}81 \\
a, c, d, f\end{array}$ & $\begin{array}{l}73 \\
a, c, d\end{array}$ & $\begin{array}{l}77 \\
a, c, d, f\end{array}$ & $85^{a, c, d, f}$ & $76^{a, c, d, f}$ \\
\hline GGALIi & $77^{d}$ & 24 & $65^{a, d}$ & $41^{f}$ & $59^{a, d}$ & 32 & 15 & 31 & 29 \\
\hline IdeC & 51 & 32 & 13 & $33^{f}$ & 12 & 34 & 23 & 70 a,c,f & 33 \\
\hline Hybrid & $88^{a, c, d}$ & $75^{a, c, d, f}$ & $\begin{array}{l}88 \\
a, c, d, f\end{array}$ & $64^{a, b, c, d, f}$ & $\begin{array}{l}85 \\
a, c, d, f\end{array}$ & $\begin{array}{l}78 \\
a, c, d\end{array}$ & $\begin{array}{l}79 \\
a, c, d, f\end{array}$ & $86^{a, c, d, f}$ & $72^{a, c, d, f}$ \\
\hline $\begin{array}{l}\text { Nutri- } \\
\text { Score }\end{array}$ & $87^{a, c, d}$ & $58^{a, c, d}$ & $60^{a, d}$ & 20 & $67^{a, d}$ & $\begin{array}{l}74 \\
a, c, d\end{array}$ & $66^{a, c, d}$ & 22 & 26 \\
\hline
\end{tabular}

Performing significantly $(p<0.05)$ better than ${ }^{a}$ : Control; b: ABIA; c: GGALIi NP; ${ }^{\mathrm{d}}$ : IdeC; e: Hybrid; f: NutriScore within the same category

If we look at the participants who correctly identified the healthier options, there appears to be no effect of education level or income. Statistical tests showed a statistically significant effect for only 3 of the 36 subgroups tested (9 food stimuli x 6 FOP label groups). Low-income participants from the control group scored significantly higher than high-income participants in selecting the healthier ice cream. Participants with a higher income who were shown the ABIA label scored significantly higher than the participants with a lower income in selecting the healthier milk drink. Finally, the less educated in the control group scored higher than the higher educated when choosing the healthier chocolate bar.

\subsection{Usefulness ratings}

Respondents were asked to rate on a scale of 0-10 how useful the label was in helping to choose the healthier product. Between $89 \%$ and $92 \%$ of respondents rated their label as either very useful (7-8) or extremely useful (9-10). Mean usefulness scores were 9.7, 9.6, 9.4, 9.6 and 9.8 for the ABIA, GGALIIi NP, IdeC, Hybrid and Nutri-Score labels, respectively, and did not differ statistically significant. The IdeC and GGALIii NP warning labels had the highest proportion (7\% and 5\%, respectively) of respondents indicating that the label was not at all useful (0-4). The proportion of respondents that rated the IdeC labels as not at all useful (7\%) was significantly higher than the $2 \%$ of respondents rating the Nutri-Score and Hybrid label as not useful. See Fig. 3 for usefulness ratings of FOP labels.

\subsection{Feedback on labels}

To better understand how the FOP labels were perceived by the respondents, they were all asked what they liked or disliked about the FOP label they were shown.

ABIA label: Most respondents said they liked the label, especially the use of colour and the clarity and objectivity of the information provided. When asked what they didn't like, some respondents indicated that 
they would also like information about other nutrients and energy.

GGALI ii NP label: Respondents were pleased with the label's simplicity, its decisive message and the fact that it attracts attention. Not all respondents were satisfied with the black colour and some were missing nutritional information.

Hybrid label: Respondents were particularly pleased with the use of the red colour as it draws attention and also the clear and easy to understand information about the nutrients and serving size. However, the language should be kept simpler (e.g. salt instead of sodium) and some respondents want more information about other nutrients and energy.

IdeC: Respondents were especially pleased with the simple information that helps people make a quick decision. Not all respondents were happy with the black colour and said it wasn't noticeable, and some would like more specific information about the amount of nutrients in the products.

Nutri-Score: When evaluating the Nutri-Score label, the respondents indicated that they liked the simple, clear message and the use of colours. Negative aspects of the Nutri-Score were the lack of information about nutrient levels and the underlying model. Some respondents misinterpreted the label as they believed that the colours and letters represented the presence of vitamins in the products.

In summary, respondents prefer simple FOP labels that use colours (not black) to convey the message. They would like to receive information about the amount of nutrients in the products, but in simple language.

\section{Discussion}

FOP labels are designed to help consumers choose healthier foods and drinks. This study compared how well five different FOP labels helped Brazilian consumers make a healthier choice between two food or drink products. It showed that the Hybrid label and the ABIA label performed best under the tested conditions. Both labels provide nutritional information per serving as well as a concise interpretation using colours (i.e. traffic light or red light only).

Most of the other studies that investigated how well different FOP labels helped consumers make a healthier choice between products, compared products within the same food category and with the same serving size, but with marked differences in nutritional profiles. These studies often found that simple interpretative labels such as Nutri-Score or warning labels were effective in helping consumers make a healthier choice [24-27]. This makes sense, because when a label clearly distinguishes between products (e.g. different score, colour or with or without a warning label), the consumer can easily make a choice. However, this does not reflect the complexity consumers face when shopping as this clear distinction does not always exist. Our research showed that when labels do not clearly distinguish between two products, or when products from different product categories or with different serving sizes are compared, these simple labels do not help the consumers to make an informed choice.

Many factors influence how consumers process information on a FOP label and how deeply this information is processed [28]. Consumers may only glance at the FOP label, process partial information or process the 
FOP label in depth. For example, the level of nutritional knowledge influences the type of information the consumer processes. Knowledgeable consumers are more likely to use the more complex nutrient information on complex labels, while a less knowledgeable consumer may look for calorie and color-coded information. Average consumers are more likely to process the information on the FOP label in depth. Under time pressure consumers will only quickly inspect the information on the FOP label and not process all available information [28]. Health-motivated consumers may also look more actively for nutritional information, while hedonically-motivated consumers may not look at nutritional information, but more at brand names [28]. So, depending on the situation, different types of FOP labels can be the most effective.

Our study showed that simple summary labels are effective when there is a clear distinction between products, but if a quick decision cannot be made, consumers will consider the nutritional information on the FOP label, when available. In those cases, interpretative labels that provide nutritional information to the consumer better assist the consumer in making an informed choice. This study showed that, even when the colours on the Hybrid and ABIA labels did not differ between products, participants could choose the healthier option based on the nutritional information presented on these labels. This suggests that the nutritional information facilitates the comparison of the nutritional content of the products, allowing the consumer to make a healthier choice. The two warning labels, i.e. IdeC and GGALI ${ }^{\mathrm{ii}}$ NP were in most cases not sensitive enough to help the consumer distinguish products based on healthiness. They did not outperform the control group. Both the Idec and GGALIii labels use very strict nutrient profiles and therefore most products bear the logo [22], making them less sensitive to distinguish products. In addition, these labels are also very simplistic and do not include additional nutritional information that can help the consumer make an informed decision where the number of sugars, saturated fats and sodium warning labels does not differ between two foods.

When labels on two different products are the same, respondents interpret this as if products are equally healthy, or respondents base their decision on other information they have about the product (e.g. packaging, type of product, claims on product, presence of other ingredients, knowledge of the brand). For example, the vegetable-oil based spread used in this study contained less saturated fat and sodium than the presented butter, so it is nutritionally, the healthier choice. However, Brazilian Dietary guidelines promote butter consumption, not vegetable-oil based spreads. Butter can therefore be seen as more natural and healthier than vegetable-oil based spreads in Brazil and this is also reflected in the results. When the FOP labels indicated that the vegetable-oil based spread was the healthier choice, most respondents chose that product. However, if no label was shown, or if the labels on butter and vegetable-oil based spreads did not differ (in case of IdeC and GGALIi NP), more than $40 \%$ of participants chose butter as the healthier option.

A recent review of FOP schemes conducted by the European Commission concluded that FOP schemes providing nutritional information per $100 \mathrm{~g}$ were better understood than portion-based schemes [13]. However, more than $90 \%$ of the food categories in Brazil have regulated serving sizes less than $100 \mathrm{~g} / 100 \mathrm{ml}$. When a nutrient profile is standard applied in $100 \mathrm{~g}$ or $100 \mathrm{~mL}$, distorted comparisons are generated. For products consumed in serving sizes $<100 \mathrm{~g}$ or $\mathrm{ml}$, the amount of nutrients to calculate the FOP label is overestimated, while for products consumed in portions of $>100 \mathrm{~g} / \mathrm{ml}$ it is underestimated. As a result, some products with small serving sizes will unfairly receive a warning label, while some products with large serving sizes that are high in nutrients of concern receive no warning label. For example, in this study, two lasagnas with a $400 \mathrm{~g}$

Page 16/25 
serving size were compared. According to the nutritional profile criteria defined by GGALIi, based on $100 \mathrm{~g}$, neither of the two products would receive a warning label, and with Nutri-Score both lasagnas would receive a score of B. These two FOP labels would thus suggest that products are healthy, despite the relatively high content of saturated fat and sodium per serving as \% GDA.

While the GGALIii NP and IdeC warning labels were the least successful in helping participants make the healthier food choice, the labels were considered by the respondents to be as useful as the other FOP labels. It is important to note that the respondents did not receive any feedback on how well they did. So they were not aware of the correct answer and how often they correctly identified the healthier option or mistakenly assumed there was no difference. One could speculate that if they got this feedback, ratings of usefulness would be lower. In any case, the ratings show that any FOP label that could help the consumer make an informed choice is considered useful by consumers. For research purposes, asking this question without providing feedback to the participants does not seem relevant. Feedback from the participants suggests that simple FOP labels that use bright colours and contain nutritional information in simple language, are liked.

Grunert et al hypothesized that consumers' liking for FOP labels is guided by three considerations: 1) consumers like simplicity, 2) when provided with simplified information consumers still want to know what it stands for and how the simplified message (e.g. warning- or health logo) has been derived, and 3) nutrition information can create a consumer resistance when they feel pushed to make choices that they do not want to take [29].

This is also confirmed by a recent study conducted by Talati et al [30] which investigated consumer perception of five FOP labels, i.e Health Star Rating, MTL, Nutri-Score, RI and a warning label. The coloured FOP labels MTL and Nutri-Score stood out and were most liked by consumers in all countries. Although the most simplified FOP labels, Nutri-Score and warning labels, were easy to understand, they were perceived as providing insufficient information and the least trusted. The RI label was perceived as the most confusing but scored high on trust. Overall, the MTL label, which combines nutrient-specific information and a summary interpretation using colour, was most liked and trusted in this study.

A strength of the current study was that it really tested the robustness of five FOP labels that differed not only in visual expression and the amount of information provided, but also in the underlying nutrient profile. Unlike other studies that mostly tested products within the same food category and with the same serving size, this study was designed to compare how well these five FOP labels enabled consumers to choose between products that differ in nutritional composition, serving size and/or food category as consumers face in real life. Another strength is that a control group has been included and that the effectiveness of the FOP labels in helping the consumer to choose the healthier product could therefore be compared with a reference group that was not given a FOP label. This provides insight into whether the presence of a specific FOP label is of added value for a consumer when making an informed choice.

Participants were also asked to select the healthier product. This demonstrates how effective the FOP label is in helping the consumer make a choice and whether it fits its purpose. In other studies consumers were asked which product they would buy [31-35], but this may be influenced by factors such as familiarity and liking of the product and the cost of the product [29]. Other studies only asked which FOP label is preferred [30, 36]. As 
demonstrated by our study, all FOP labels were rated as very useful, irrespective of their efficacy in helping the consumer choose the healthier option. Only asking for preference is thus not very useful.

This study also has some limitations. The participants were a representative sample of the Brazilian population. Therefore, we also included participants with a lower education level, who may have had difficulty understanding the information on the FOP labels. However, socio-economic status and level of education were similar between the six FOP label groups and therefore we did not expect this to affect the outcomes of the study. This was confirmed by statistical subgroup analyses that showed that participants who correctly identified the healthier options, did not differ in education level or income. Another limitation is that we did not ask participants if they were colour-blind. Thus, it is possible that participants with colourblindness were included, which may have adversely affected the ability to understand the colour-coded labels. However, none of the participants who were shown the Abia or Nutri-Score labels, voluntarily reported to be colour-blind and thus unable to interpret the labels.

The FOP labels we tested in this online survey were selected because they were under consideration by Anvisa, the National Health Surveillance Agency of Brazil, at the time we designed this study. Anvisa proposed in its preliminary report on the regulatory impact analysis on nutrition labelling [22] to focus only on the three nutrients of concern, sugar, saturated fat and sodium. We therefore decided to only use the content of these three nutrients to inform the different FOP labels (with exception of Nutri-Score). Focusing on just these three nutrients of concern is a limitation to assessing the healthiness of a product.

Brazilian regulations do not require the sugar content of food products to be stated on the packaging. For some of the products used in this study (ice creams, frozen meals, dairy alternative drink, sweet snacks), we had to estimate the sugar content. These estimates were unlikely to deviate very much from the actual sugar content and were used for all FOP labels.

This study was conducted online using pictures of actual products. It does therefore not reflect a real-life situation in which participants can examine packaging and other information, such as the nutrition table on the back, to make an informed choice. Finkelstein et al [37] attempted to mimic a real-life situation by asking the participants $(n=147)$ to purchase their weekly groceries in an online grocery store with 3343 foods and 832 beverages. Participants only had access to back-of-pack Nutrition Information Tables or were also shown an MTL label or Nutri-Score label. Both the MTL and Nutri-Score FOP labels improved the dietary quality of the purchases as compared to the control group. The Nutri-Score label performed best in improving overall diet quality, but unlike Nutri-Score, the MTL label reduced calories. Thus, FOP labels had added value when purchasing products, even in the presence of a Nutrition Information Table.

\section{Conclusions}

In conclusion, this study showed that the Hybrid and the ABIA FOP labels, two interpretative labels that use colours and provide nutritional information per serving, were best suited to help Brazilian consumers choose the healthier product. The other three labels are based on per $100 \mathrm{~g}$ and therefore will not always discriminate enough to help consumers. The ABIA and Hybrid labels outperformed the other FOP labels when serving 
sizes differed significantly or when deeper consideration of nutritional information was needed to make an informed decision.

\section{Abbreviations}

ABIA

Associação Brasileira das Indústrias da Alimentação

ENL

Evolved Nutrition Label

FOP

Front-of-pack

GDA

Guideline Daily Amounts

GGALI

Gerência-Geral de Alimentos

IDEC

Instituto Brasileiro de Defesa do Consumidor

MTL

Multiple Traffic Light

PAHO

Pan American Health Organization

RI

Reference Intake

WHO

World Health Organization

\section{Declarations}

\section{Ethics approval and consent to participate}

Not applicable. The current study is not considered to be medical research and is therefore exempted from medical ethical review. All participants provided written consent for the collection and use of their data for research purposes when subscribing to the research panel. All participants voluntarily participated in this online survey.

\section{Consent for publication}

Not applicable.

\section{Availability of data and materials}

The datasets used and/or analysed during the current study are available from the corresponding author on reasonable request. 


\section{Competing interests}

The authors, Wendy Blom, Chantal Goenee, Els de Groene and Fernanda de Oliveira Martins were all employed by Unilever, the sponsor of this study, when the study was performed. Lucia Juliano declares no conflict of interest.

\section{Funding}

This research was funded by Unilever. WAMB, CG, EMdG and FdOM were employed by Unilever when this research was conducted.

\section{Authors' contributions}

Conceptualization, CG and EMdG; Data curation and Formal analysis, LJ; Methodology, CG, LJ, EMdG and FdOM; Writing - original draft, WAMB; Writing - review \& editing, CG, LJ, EMdG and FdOM. All authors have read and agreed to the published version of the manuscript.

\section{Acknowledgements}

We thank the volunteers who participated in this online survey and all other staff at Harris Interactive, Toluna and Unilever who helped with the study or provided input to this manuscript.

\section{References}

1. Cowburn G, Stockley L. Consumer understanding and use of nutrition labelling: a systematic review. Public Health Nutr. 2005;8(1):21-8. doi:10.1079/phn2005666.

2. World Health Organization. Guiding principles and framework manual for front-of-pack labelling for promoting healthy diets 2019. World Health Organization, Geneva.

3. Kanter R, Vanderlee L, Vandevijvere S. Front-of-package nutrition labelling policy: global progress and future directions. Public Health Nutr. 2018;21(8):1399-408. doi:10.1017/S1368980018000010.

4. Labonté M-Ė, Poon T, Gladanac B, Ahmed M, Franco-Arellano B, Rayner M, L'Abbé MR. Nutrient Profile Models with Applications in Government-Led Nutrition Policies Aimed at Health Promotion and Noncommunicable Disease Prevention: A Systematic Review. Advances in Nutrition. 2018;9(6):741-88. doi:10.1093/advances/nmy045.

5. van der Bend DLM, Lissner L. Differences and Similarities between Front-of-Pack Nutrition Labels in Europe: A Comparison of Functional and Visual Aspects. Nutrients(2019;11(3). doi:10.3390/nu11030626.

6. The Food and Drink Federation. Food labelling. 2020. https://www.gdalabel.org.uk/gda/referenceintakes.html. 
7. Food Standards Agency. Front of Pack nutrition labelling guidance. 2020.

https://www.gov.uk/government/publications/front-of-pack-nutrition-labelling-guidance. Accessed 25 February 2020.

8. Swedish National Food Agency The Keyhole. https://www.livsmedelsverket.se/en/food-andcontent/labelling/nyckelhalet. Accessed 25 February 2020.

9. Choices International Foundation Choices programme. https://www.choicesprogramme.org. Accessed 25 February 2020.

10. Australian Government of Health Front-of-pack labelling updates. https://foodregulation.gov.au/internet/fr/publishing.nsf/Content/front-of-pack-labelling-1. Accessed 25 February 2020.

11. Julia CH. S. Development of a new front-of-pack nutrition label in France: the five-colour Nutri-Score. Public Health Panorama. 2017;3(4):712-25.

12. Reyes M, Garmendia ML, Olivares S, Aqueveque C, Zacarias I, Corvalan C. Development of the Chilean front-of-package food warning label. BMC Public Health. 2019;19(1):906. doi:10.1186/s12889-019-71181.

13. Storksdieck genannt Bonsmann SM, Cirolo G, van Bavel E, Wollgast R. J. Front-of-pack nutrition labelling schemes: a comprehensive review. Luxembourg: Publications Office of the European Union; 2020. doi:10.2760/436998.

14. Cecchini M, Warin L. Impact of food labelling systems on food choices and eating behaviours: a systematic review and meta-analysis of randomized studies. Obes Rev. 2016;17(3):201-10. doi:10.1111/obr.12364.

15. Temple NJ. Front-of-package food labels: A narrative review. Appetite. 2020;144:104485. doi:https://doi.org/10.1016/j.appet.2019.104485.

16. Federal public service Health Food Chain Safety and Environment Belgium. Nutri-Score. 2019. https://www.health.belgium.be/nl/de-nutri-score-0. Accessed 3 March 2020.

17. Bundesministerium für Ernährung und Landwirtschaft. Ergebnis der Verbraucherbeteiligung liegt vor: Bundesministerin Julia Klöckner wird Nutri-Score(R) einfüren. 2019. https://www.bmel.de/SharedDocs/Pressemitteilungen/2019/197-eNWK.html.

18. Cox Vásconez AE. RESOLUCIÓN No. 14 511, 2014. Gobierno Nacional de la Republica del Ecuador. Ministerio de Industrias Y Productividad. Subsecretaria de la Calidad. Quito, Ecuador.

19. Carreño I. Chile's Black STOP Sign for Foods High in Fat, Salt or Sugar. European Journal of Risk Regulation. 2015;6(4):622-8. doi:10.1017/S1867299X0000516X.

20. El Presidente de la Republica Peru. Aprueban Manual de Advertencias Publicitarias en el marco de lo establecido en la Ley No 30021, Ley de promoción de la alimentación saludable para niños, niñas y adolescentes, y su Reglamento aprobado por Decreto Supremo Nº17-2017-SA. Peru: El Peruano; 2018.

21. Rojo Sánchez AGNB. JA. Modificación a la Norma Oficial Mexicana NOM-051-SCFI/SSA1-2010, Especificaciones generales de etiquetado para alimentos y bebidas no alcohólicas preenvasadosInformación comercial y sanitaria, publicada el 5 de abril de 2010. vol NOM-051-SCFI/SSA1-2010. Mexico City. 2020. 
22. Agência Nacional de Vigilancia Sanitária (Anvisa). Relatório Preliminar de Análise de Impacto Regulatório sobre Rotulagem Nutricional. Brasília: Gerência-Geral de Alimentos; 2018.

23. Egnell M, Kesse-Guyot E, Galan P, Touvier M, Rayner M, Jewell J, Breda J, Hercberg S, Julia C. Impact of Front-of-Pack Nutrition Labels on Portion Size Selection: An Experimental Study in a French Cohort. Nutrients. 2018;10(9). doi:10.3390/nu10091268.

24. Egnell M, Ducrot $P$, Touvier M, Alles B, Hercberg S, Kesse-Guyot E, Julia C. Objective understanding of Nutri-Score Front-Of-Package nutrition label according to individual characteristics of subjects: Comparisons with other format labels. PloS one. 2018;13(8):e0202095. doi:10.1371/journal.pone.0202095.

25. Egnell M, Talati Z, Gombaud M, Galan P, Hercberg S, Pettigrew S, Julia C. Consumers' Responses to Front-of-Pack Nutrition Labelling: Results from a Sample from The Netherlands. Nutrients. 2019;11(8). doi:10.3390/nu11081817.

26. Galan P, Egnell M, Salas-Salvado J, Babio N, Pettigrew S, Hercberg S, Julia C. Understanding of different front-of-package labels by the Spanish population: Results of a comparative study. Endocrinologia diabetes y nutricion. 2019. doi:10.1016/j.endinu.2019.03.013.

27. Arrua A, Machin L, Curutchet MR, Martinez J, Antunez L, Alcaire F, Gimenez A, Ares G. Warnings as a directive front-of-pack nutrition labelling scheme: comparison with the Guideline Daily Amount and traffic-light systems. Public Health Nutr. 2017;20(13):2308-17. doi:10.1017/S1368980017000866.

28. Sanjari SS, Jahn S, Boztug Y. Dual-process theory and consumer response to front-of-package nutrition label formats. Nutrition reviews. 2017;75(11):871-82. doi:10.1093/nutrit/nux043.

29. Grunert KG, Wills JM. A review of European research on consumer response to nutrition information on food labels. J Public Health. 2007;15(5):385-99. doi:10.1007/s10389-007-0101-9.

30. Talati Z, Egnell M, Hercberg S, Julia C, Pettigrew S. Consumers' Perceptions of Five Front-of-Package Nutrition Labels: An Experimental Study Across 12 Countries. Nutrients. 2019;11(8):1934. doi:10.3390/nu11081934.

31. Talati Z, Egnell M, Hercberg S, Julia C, Pettigrew S. Food Choice Under Five Front-of-Package Nutrition Label Conditions: An Experimental Study Across 12 Countries. American journal of public health. 2019. doi:10.2105/ajph.2019.305319.

32. Mora-Garcia CA, Tobar LF, Young JC. The Effect of Randomly Providing Nutri-Score Information on Actual Purchases in Colombia. Nutrients. 2019;11(3). doi:10.3390/nu11030491.

33. Billich N, Blake MR, Backholer K, Cobcroft M, Li V, Peeters A. The effect of sugar-sweetened beverage front-of-pack labels on drink selection, health knowledge and awareness: An online randomised controlled trial. Appetite. 2018;128:233-41. doi:10.1016/j.appet.2018.05.149.

34. Machin L, Arrua A, Gimenez A, Curutchet MR, Martinez J, Ares G. Can nutritional information modify purchase of ultra-processed products? Results from a simulated online shopping experiment. Public Health Nutr. 2018;21(1):49-57. doi:10.1017/S1368980017001185.

35. Acton RB, Jones AC, Kirkpatrick SI, Roberto CA, Hammond D. Taxes and front-of-package labels improve the healthiness of beverage and snack purchases: a randomized experimental marketplace. Int J Behav Nutr Phys Act. 2019;16(1):46. doi:10.1186/s12966-019-0799-0. 
36. Dana LM, Chapman K, Talati Z, Kelly B, Dixon H, Miller C, Pettigrew S. Consumers' Views on the Importance of Specific Front-of-Pack Nutrition Information: A Latent Profile Analysis. Nutrients. 2019;11(5). doi:10.3390/nu11051158.

37. Finkelstein EA, Ang FJL, Doble B, Wong WHM, van Dam RM. A Randomized Controlled Trial Evaluating the Relative Effectiveness of the Multiple Traffic Light and Nutri-Score Front of Package Nutrition Labels. Nutrients. 2019;11(9). doi:10.3390/nu11092236.

\section{Figures}
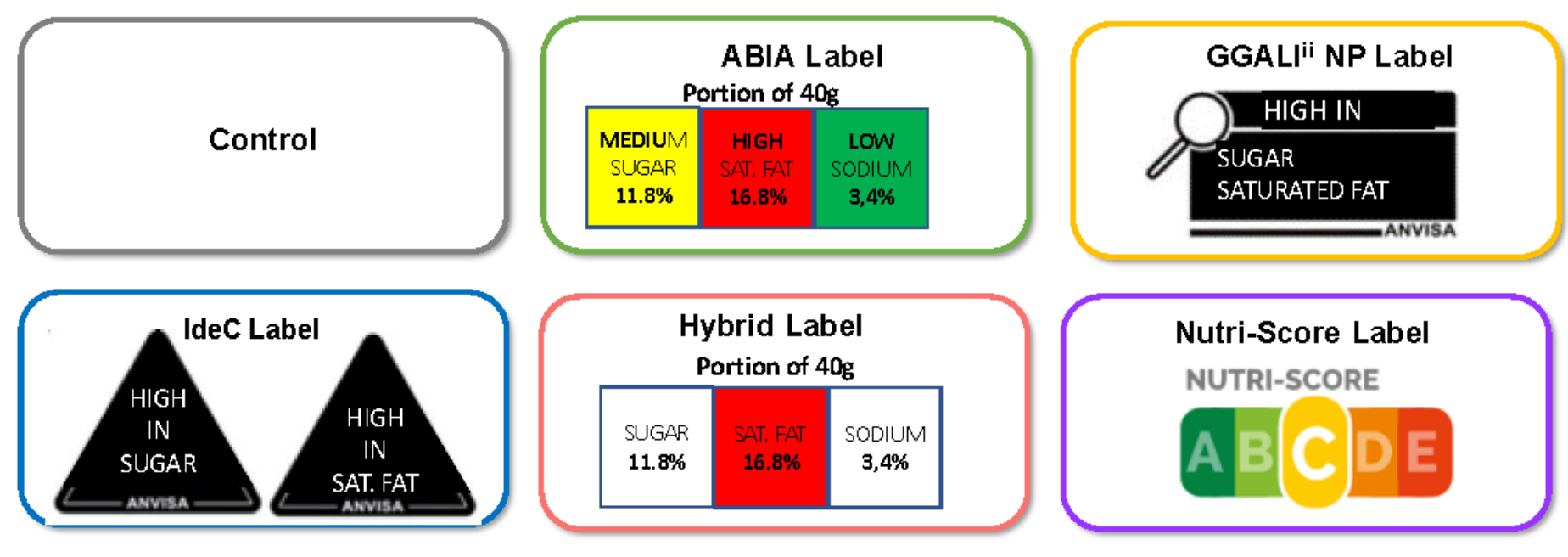

\section{Figure 1}

Example of front-of-pack labels used in this study (translated from Portuguese to English) 


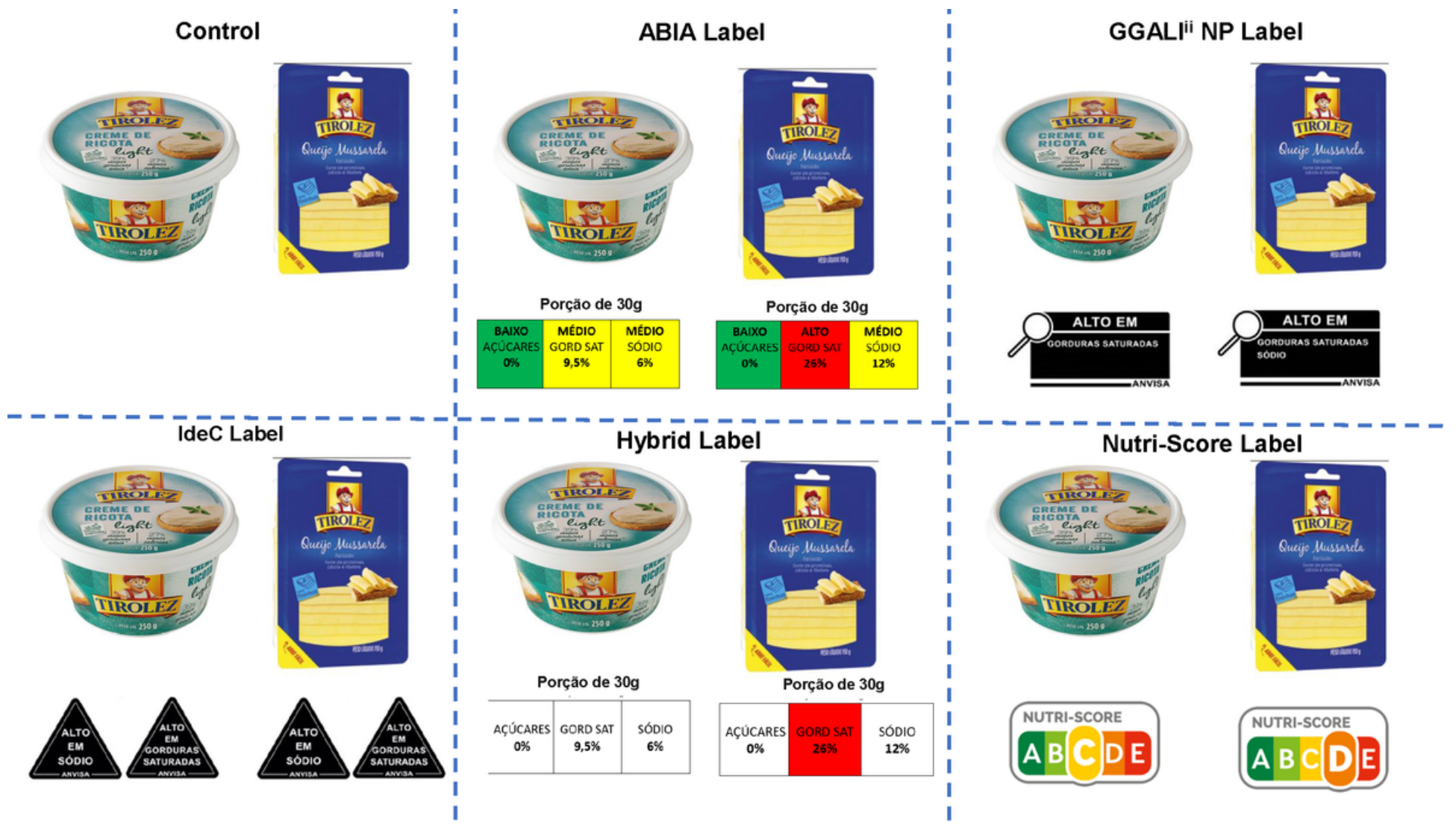

Figure 2

Example of stimulus used for each randomized group. Legend Source pictures: www.shoppr.com.br (Crème de Ricota Light) and www.tirolez.com (Queijo Mussarela)

$7-10$ score

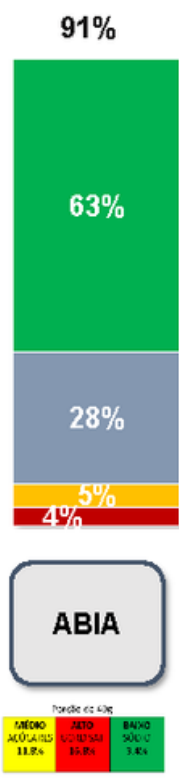

$90 \%$
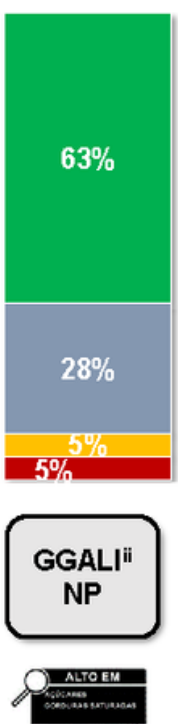

$89 \%$

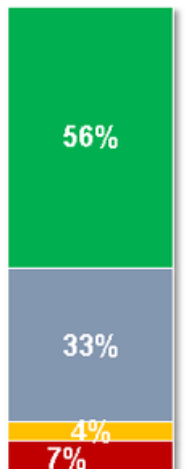

$7 \%$

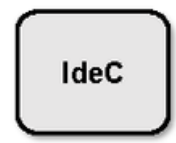

And
$92 \%$

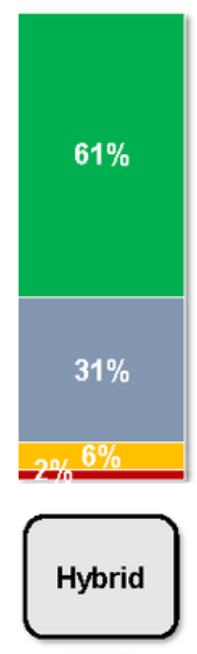

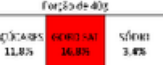

$92 \%$

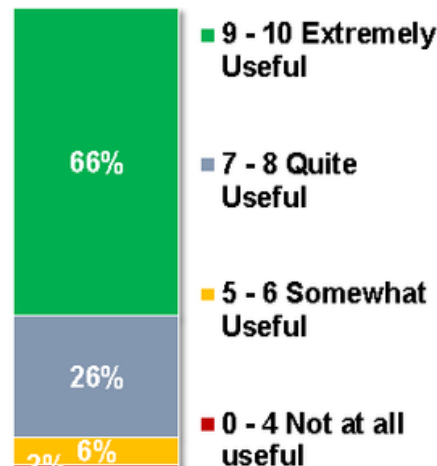

Nutri-

Score 


\section{Figure 3}

Usefulness ratings of the five FOP label. Footnote Answer to Question: : To what extent is the label below useful when helping you make healthy food choices? 